\title{
Effect of core strengthening with pelvic proprioceptive neuromuscular facilitation on trunk, balance, gait, and function in chronic stroke
}

\author{
Vishal Sharma', Jaskirat Kaur ${ }^{2, *}$ \\ 1Physiotherapy Department, School of Medical and Allied Sciences, Galgotias University, Gautam Buddh Nagar, Uttar Pradesh, India \\ ${ }^{2}$ Physiotherapy Department, Indian Spinal Injuries Centre Institute of Rehabilitation Sciences, New Delhi, India
}

The purpose of this study was to evaluate the effects of core strengthening combined with pelvic proprioceptive neuromuscular facilitation (PNF) on trunk impairment, balance, gait, and functional ability of chronic stroke patients. Twenty-three participants with chronic stroke were recruited and randomly allocated to one of the two groups: core strengthening combined with pelvic PNF (group 1, $n=13$ ), and pelvic PNF with trunk flexibility exercises (group $2, n=10$ ). Intervention was given to both groups for 60 min per session 5 times per week for 4 weeks. Performance of both groups was evaluated on Trunk Impairment Scale, Tinetti Performance Oriented Mobility Assessment (Tinetti-POMA), Balance Evaluation Systems Test (Mini-BESTest), Wisconsin
Gait Scale, and Barthel Activities of Daily Living Index prior to and after the completion of the intervention. The comparison between postintervention scores of Tinetti-POMA between group $1(18.76 \pm 1.78)$ and group $2(16.8 \pm 1.87)$ and Mini-BESTest group $1(16.15 \pm 1.28)$ and group 2 $(14.7 \pm 1.41)$ showed significant difference $(P=0.018)$. The results indicated that core stabilisation combined with pelvic PNF was more effective for improving trunk impairment, balance and gait of chronic stroke patients.

Keywords: Stroke, Core strengthening, Proprioceptive neuromuscular facilitation, Balance, Gait

\section{INTRODUCTION}

Stroke is a global health care problem leading to significant mortality and morbidity (Langhorne et al., 2011). In India the prevalence of stroke is estimated to be 203 per 100,000 people and it is projected to rank as the fourth leading cause of disability by the year 2020 (Taylor and Kumar, 2012). The deficits resulting have significant impact on independence, quality of life and productivity of the survivors.

Common deficiencies in stroke include spasticity, weakness, and loss of equilibrium on the affected side causing inability to maintain postural alignment (Pathak et al., 2015). The trunk is considered as a central key point to allow the body to remain upright and adjust weight shifts during static and dynamic postural adjustments (Zakaria et al., 2010). Following stroke one side of the limbs are affected but trunk muscles are affected on both the sides leading to insufficient trunk rotation, difficulty in maintaining balance and gait (Shinde and Ganvir, 2014).

Pelvic proprioceptive neuromuscular facilitation (PNF) helps to improve control of pelvis which is a key point for maintaining trunk control, gait and balance (Wang, 1994) by stimulation of muscle and joint proprioception. Studies in the past have shown a significant correlation between pelvic tilt and trunk control across various stages of stroke (Zakaria et al., 2010).

Core musculature include muscles of the trunk and pelvis that are responsible for maintaining stability of spine and pelvis (Kibler et al., 2006). The abdominals in front, paraspinals and gluteals at the back, the diaphragm as the roof and pelvic and hip
${ }^{*}$ Corresponding author: Jaskirat Kaur (iD http://orcid.org/0000-0002-6755-9146 Physiotherapy Department, Indian Spinal Injuries Centre Institute of Rehabilitation Sciences Sector C, Vasant Kunj, New Delhi 110070, India Tel: +91-9891264977, Fax: +91-11-26894804, E-mail: drjaskirat24@gmail.com Received: January 5, 2017 / Accepted: March 31, 2017
This is an Open Access article distributed under the terms of the Creative Commons Attribution Non-Commercial License (http://creativecommons.org/licenses/by-nc/4.0/) which permits unrestricted non-commercial use, distribution, and reproduction in any medium, provided the original work is properly cited. 
girdle musculature as the bottom constitute the core musculature (Shinkle et al., 2012). Previous studies have shown that central nervous system initiates contraction of the abdominal muscles and multifidus in a feed-forward manner in advance of the prime mover of lower limb to stabilize the spine. Transverse abdominis is the first muscle that is active during movement of a lower limbs following contralateral weight shifting (Hodges and Richardson, 1997). These muscles have been used for training of athletes and patients with low back pain (Akuthota and Nadler, 2004). Studies have found that core training improves static and dynamic balance in chronic stroke patients (Chung et al., 2013). We hypothesize that addition of core stabilization program to pelvic PNF would help in improving core stability in order to attain trunk control and controlled mobility for improving balance, gait and functional ability in stroke patients.

\section{MATERIALS AND METHODS}

\section{Participants and design}

An assessor-blinded randomised controlled trail was undertaken. Random sampling was done based on the inclusion and exclusion criteria. The distribution of sample and sample size is explained in CONSORT (consolidated standards for reporting of trials) flowchart (Fig. 1). Participants were recruited from Rehabilitation Department of Indian Spinal Injuries Hospital, Vasant Kunj and Physiotherapy Department, Vidyasagar Institute of Mental Health, Neurology and Allied Sciences, Delhi. Participants with first ever unilateral ischemic stroke involving middle cerebral artery territory; duration of stroke more than 6 months; age between 45-60 years were included. Participants should be able to walk with or without support for $10 \mathrm{~m}$. Participants should be able to understand and follow simple verbal instructions (Mini-Mental Status Examination [MMSE] $\geq 24$ ). Participants with recurrent stroke; brainstem or cerebellar stroke or haemorrhagic stroke were excluded. Also, participants with severe spasticity (modified ashworth scale grade $\geq 3$ ) or severe flaccidity in lower limbs and upper limbs were excluded.

\section{Procedure}

The participants included signed an informed consent form. For random allocation a computer generated random allocation schedule was created by a person other than the principal investigator. To ensure concealment the allocation schedule was sequentially numbered and sealed in opaque envelopes. Person not associated with the study opened the numbered envelopes sequentially to reveal the participant's group allocation. The two groups

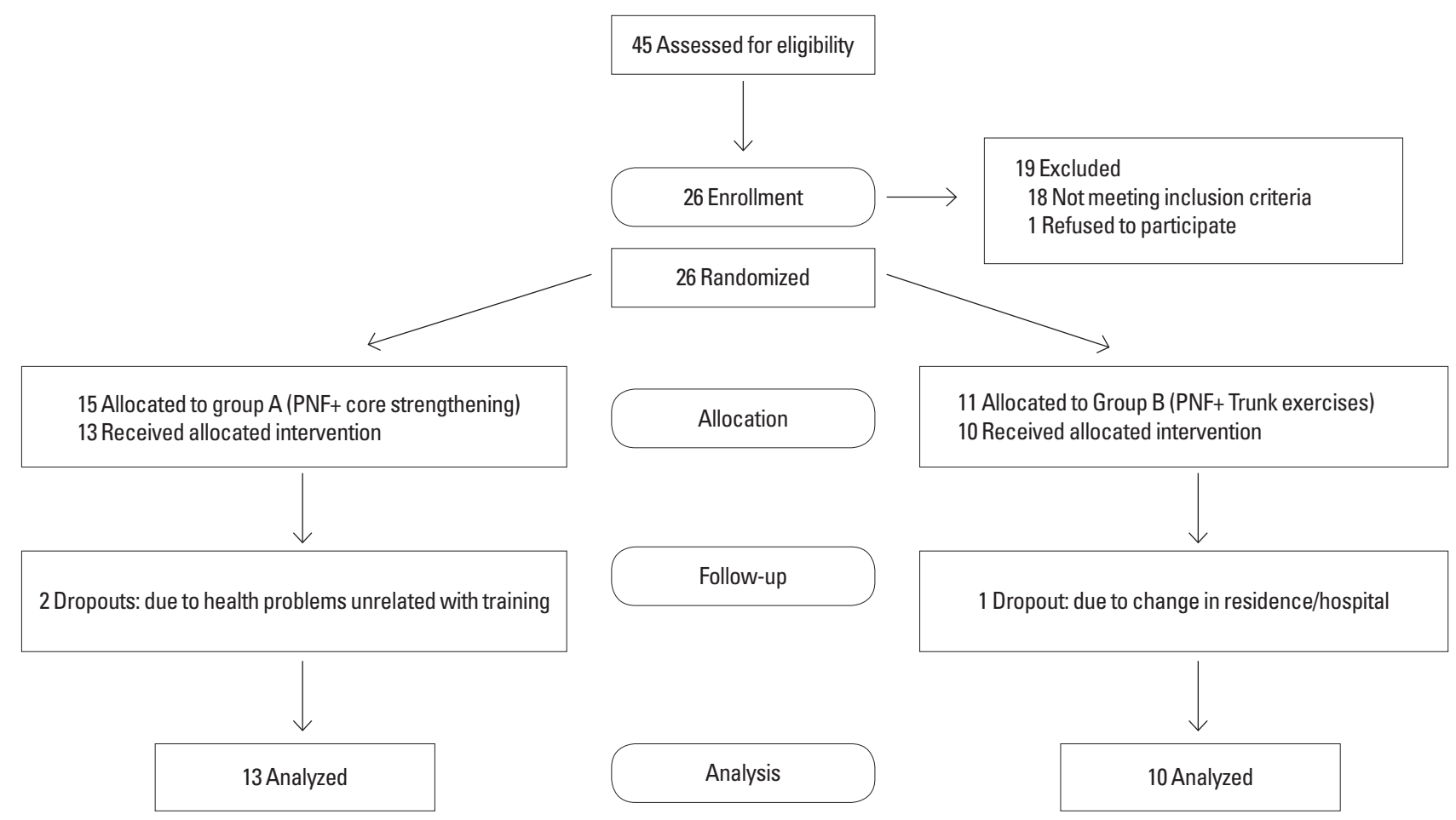

Fig. 1. Consolidated standards for reporting of trials (CONSORT) diagram. 
were group 1 - pelvic PNF + core strengthening group and group 2-pelvic PNF. The study was approved by the Institutional Research Review Committee and the Institutional Ethical Committee (ISIC/IIRS/RP/2015/073). We certify that all applicable institute and government registration concerning the ethic use of human volunteers were followed during the course of this research.

\section{Procedure for group 1 \\ Core stabilization}

Participants in group 1 were positioned in supine. They were asked to recognize their neutral spine position that is midrange between flexion and extension. The core muscles trained were transverse abdominis, multifidus, paraspinals, quadratus lumborum, and obliques. In the first stage the participants were taught to activate abdominal wall musculature. They were initially trained to perform abdominal bracing. In order to ensure that the participants were contracting the right musculature a pressure biofeedback device was used. The lower end of inflatable bag was placed at the posterior superior iliac spine. Before starting the contraction, the bag was inflated to a pressure of $40 \mathrm{mmHg}$ with the valve closed and participants were instructed to breathe deeply using mainly abdominal wall musculature, then the inflatable bag was adjusted to $40 \mathrm{mmHg}$ again. Participants were requested to perform abdominal muscle contractions with following verbal commands standardized by examiner: "Tighten your abdomen in order to make it like a rigid cylinder without moving your ribs and pelvis". Since the contraction of transverse abdominis results in an increase in pressure ranging from $4-10 \mathrm{mmHg}$, hence a pressure rise of at least $4 \mathrm{mmHg}$ was considered as positive reinforcement for correct movement. Once the patient mastered the technique of abdominal bracing progression was made to other core stability exercises. Participants were then positioned in quadruped position and asked to lift alternate arms, gradually progressing to alternate leg lifts and alternate arm/leg raises to activate multifidus. This was followed by side bridges (side plank) exercise for activation of quadratus lumborum and obliques (Akuthota et al., 2008). The participants were then asked to perform trunk curls in crook lying, asked to lift their upper trunk slightly $\left(15^{\circ}\right)$ from the plinth, hold the position for $5 \mathrm{sec}$. The progression of exercises was done once the patient was able to perform 30 repetition of each exercise with 8-sec hold. The participants were told to maintain normal diaphragmatic breathing throughout the intervention.

\section{Proprioceptive neuromuscular facilitation}

Patterns of movement performed were anterior elevation - poste- rior depression of the hemiplegic side. Participants' were positioned in side lying on the unaffected side, with both hips flexed to $100^{\circ}$ and the knees flexed to $45^{\circ}$. Their neck was supported by a pillow and flexed to $30^{\circ}$. The therapist stood behind the participants facing the direction of pelvic movement of the subject. Therapist's hands were placed on anterior iliac spine of the subject's pelvis for anterior elevation or on the subject's ischial tuberosity for posterior depression. Stretch was applied immediately and gently after the target muscles had been fully lengthened but before the subject started to move. Resistance was applied variably to obtain a smooth and coordinated movement. Assistance was provided if required by the participants. Specific and timed commands were given to obtain the desired movement. "Pull up" was used to facilitate pelvic anterior elevation, and "push down" and "sit into my hands" were used to facilitate pelvic posterior depression (Wang, 1994).

The elements of PNF such as positioning, manual contact, resistance and verbal commands were incorporated into the treatment. Techniques used in this study were rhythmic initiation, slow reversal, and agonistic reversals. The sequence was rhythmic initiation first for $10 \mathrm{~min}$, then slow reversal for $10 \mathrm{~min}$, and then agonistic reversals for an additional 10 min with 2 min of rest between each technique. A stopwatch was used to measure the time.

\section{Procedure for group 2}

Participants in group 2 received pelvic PNF along with trunk flexibility exercises, which consisted task-specific exercises of the upper and lower part of the trunk. The exercises were performed both in the supine and in sitting positions (Verheyden et al., 2009). It was performed for total of 30 min including 6 min of rest period in between as per the patients requirement, once in a day, 5 days per week for 4 weeks. Procedure followed for PNF was similar to group 1.

Intervention was given for 60 min per session 5 times per week for 4 weeks for both groups.

\section{Outcome measures}

The pre- and postintervention scores of Trunk Impairment Scale (TIS), Tinetti Performance Oriented Mobility Assessment (Tinetti-POMA), Balance Evaluation Systems Test (Mini-BES Test), Wisconsin Gait Scale (WGS) and Barthel Activities of Daily Living Index (BI) were evaluated for both groups.

\section{Data analysis}

Data was analysed using IBM SPSS Statistics ver. 21.0 (IBM Co., Armonk, NY, USA). Sample size was determined through 
power calculation based on previous studies fore core stabilization in stroke patients with an estimated effect size of 0.80 an overall sample of 16 participants ( 8 in each group) at 0.05 level of significance was estimated. However, 26 subjects were recruited to allow $10 \%$ dropout. Mean change score were calculated as the difference between post- and pretest scores and an independent $t$-test was used to test the difference in the changed scores between two groups. Paired $t$-test was used to analyze within group differences. A level of significance was set at 0.05 .

\section{RESULTS}

\section{Demographic details of participants}

Group 1 included 13 participants (male, 11; female, 2) with mean age $57 \pm 8.25$ years; mean weight $66.9 \pm 6.04 \mathrm{~kg}$; mean height $172.40 \pm 3.30 \mathrm{~cm}$; chronicity of stroke $13.3 \pm 4.27$ months, and MMSE scores of $26.30 \pm 1.70$. Group 2 consisted of 10 participants (male, 9; female, 1 ) mean age $57.23 \pm 7.39$ years; mean weight $71.30 \pm 9.23 \mathrm{~kg}$; mean height $173.84 \pm 5.32 \mathrm{~cm}$, chronicity of stroke $12.15 \pm 3.89$ months, and MMSE score of 25.46 \pm 1.45. Baseline comparison between groups is explained in Table 1.

Table 1. Demographic details of participants

\begin{tabular}{lccc}
\hline Variable & Group 1 $(\mathrm{n}=13)$ & Group 2 $(\mathrm{n}=10)$ & $P$-value \\
\hline Age $(\mathrm{yr})$ & $57.23 \pm 7.39$ & $57 \pm 8.26$ & 0.944 \\
Height $(\mathrm{cm})$ & $173.84 \pm 5.32$ & $172.40 \pm 3.30$ & 0.463 \\
Weight $(\mathrm{kg})$ & $71.30 \pm 9.23$ & $66.90 \pm 6.04$ & 0.183 \\
Time since stroke $(\mathrm{mo})$ & $12.15 \pm 3.89$ & $13.30 \pm 4.27$ & 0.509 \\
Side of weakness, right:left & 8.5 & $6: 4$ & \\
MMSE & $25.46 \pm 1.45$ & $26.30 \pm 1.70$ & 0.216 \\
\hline
\end{tabular}

Values are presented as mean \pm standard deviation or number. Group 1, core strengthening+proprioceptive neuromuscular facilitation group; group 2, core strengthening group; MMSE, Mini-Mental Status Examination.

$P \leq 0.05$, statistically significant.

\section{Within group analysis}

The pre- and postintervention scores of within group 1 showed significant improvements on TIS scores $(t=-10.143, P=0.001)$; Tinetti-POMA scores $(t=-16.915, P=0.001)$; Mini-BESTest scores $(t=-18.403, P=0.001)$; WGS scores $(t=-20.529, P=$ $0.001)$. The comparison of preintervention $(14.0 \pm 1.96)$ and postintervention $(14.7 \pm 2.09)$ scores of $B I$ within group 1 showed significant difference $(t=-3.32, P=0.006)$.

The comparison of preintervention $(10.90 \pm 2.07)$ and postintervention $(14.0 \pm 1.15)$ scores of TIS within group 2 showed statistically significant difference $(P=0.001)$. The comparison of pre intervention $(14.23 \pm 1.53)$ and postintervention $(14.7 \pm 1.41)$ scores of Tinetti-POMA within group 2 showed statically significant difference $(t=-4.16, P=0.002)$. The comparison of preintervention $(10.6 \pm 1.34)$ and postintervention $(14.7 \pm 1.41)$ scores of Mini-BESTest within group 2 showed significant difference $(P=0.001)$. The comparison of pre intervention $(26.10 \pm 2.32)$ and postintervention $(22.01 \pm 2.47)$ scores of WGS within group 2 showed significant difference $(P=0.001)$. The comparison of preintervention $(14.4 \pm 1.95)$ and postintervention $(15.1 \pm 2.13)$ scores of BI within group 2 showed significant difference $(P=$ $0.025)$. The comparison between mean change in scores between groups 1 and 2 are depicted in Table 2 .

\section{DISCUSSION}

As postinjury symptoms, stroke patients have several deficits including impaired motor control, difficulty with co-ordination and postural adjustments, and difficulty in maintaining balance and gait. In this study we added stabilization of core musculature to pelvic PNF techniques. Within group analysis show a significant improvement in the scores of TIS, Tinetti-POMA, MiniBESTest, WGS, and BI for pelvic PNF group and core along with

Table 2. Comparison of scores of groups 1 and 2

\begin{tabular}{|c|c|c|c|c|c|c|c|c|}
\hline \multirow{2}{*}{ Variable } & \multicolumn{2}{|c|}{ Group 1} & \multicolumn{2}{|c|}{ Group 2} & \multicolumn{2}{|c|}{ Mean change scores } & \multirow{2}{*}{$t$-value } & \multirow{2}{*}{$P$-value } \\
\hline & Preintervention & Postintervention & Preintervention & Postintervention & Group 1 & Group 2 & & \\
\hline TIS & $10.92 \pm 1.25$ & $15.46 \pm 1.39$ & $10.90 \pm 2.07$ & $14.0 \pm 1.15$ & 0.23 & 1.46 & $2.683^{*}$ & 0.014 \\
\hline Tinetti-POMA & $14.23 \pm 1.53$ & $18.76 \pm 1.78$ & $14.7 \pm 1.41$ & $16.8 \pm 1.87$ & -0.46 & 1.90 & $2.566^{*}$ & 0.018 \\
\hline Mini-BESTest & $9.69 \pm 1.18$ & $16.15 \pm 1.28$ & $10.6 \pm 1.34$ & $14.7 \pm 1.41$ & -0.90 & 1.45 & $2.577^{*}$ & 0.018 \\
\hline WGS & $27.01 \pm 2.13$ & $20.0 \pm 1.62$ & $26.10 \pm 2.32$ & $22.01 \pm 2.47$ & 0.91 & -2.01 & $2.356^{*}$ & 0.028 \\
\hline $\mathrm{Bl}$ & $14.0 \pm 1.96$ & $14.7 \pm 2.09$ & $14.4 \pm 1.95$ & $15.1 \pm 2.13$ & -0.40 & -0.41 & -0.459 & 0.650 \\
\hline
\end{tabular}

Values are presented as mean \pm standard deviation.

Group 1, core strengthening+proprioceptive neuromuscular facilitation group; group 2, core strengthening group; TIS, Trunk Impairment Scale; Mini-BESTest, Mini-Balance Evaluation Systems Test; WGS, Winconsin Gait Scale; BI, Barthel Index.

${ }^{*} P \leq 0.05$, statistically significant. 
pelvic PNF group. Pelvic PNF techniques act by facilitating the neuromuscular mechanism through application of stretch, use of particular movement patterns and use of maximal resistance in order to induce irradiation. The results are consistent with previous studies where PNF has been used to improve trunk control, gait and balance. Kumar et al. (2012) evaluated the effects of PNF technique on gait parameters and functional mobility in hemiparetic with post stroke duration of less than 6 months for $30 \mathrm{~min}$ daily, 3 days a week for 4 weeks and found that pelvic PNF has significant effect on gait parameters and functional mobility in hemiparetic patients (Kumar et al., 2012).

In another study by Shinde and Ganvir (2014) evaluated the effectiveness of trunk PNF pattern on improving the trunk balance in acute stroke patients and found significant improvement in balance and trunk performance in acute stroke patients (Shinde and Ganvir, 2014).

Another randomized control trial was conducted to evaluated the effects of PNF on gait and found significant improvement in the WGS scores from 0 to 4 weeks and the scores not much significantly different post 6 weeks of assessment, indicating that the improvements gained during the 4 weeks of treatment are sustained even after cessation of the intervention. The studies suggest that these facilitatory techniques might help to facilitate trunk motion and pelvic stability thus enhancing the motor control (Mann et al., 2013).

Pelvis provides base of support for core musculature. Core musculature includes the muscles of trunk and pelvis that are responsible for the maintenance of stability of spine and pelvis and help in generation and transfer of energy from large to small body parts during many activities like walking, running. Core strength is an integral component of the complex phenomena that comprise balance, which requires a multidimensional interplay between central, peripheral, sensory, and motor systems. Training the domain of balance is important for functional movements.

Core acts as an anatomical basis for motion of distal segment. Core stability involves the ability to control motion of trunk over pelvis and legs in order to maintain stability of spine (Kibler et al., 2006). It was assumed that addition of core stability would provide more stability to improve to allow optimum force production and control of movement of lower legs.

\section{Improvement in balance scores}

Balance is a complex process involving the reception and integration of sensory inputs and the planning and execution of movement, to achieve a goal requiring upright posture.

Stroke can affect the various systems involved in postural con- trol or the way these systems work together. Weakness on one side of body, loss of sensation in affected side, particularly leg, injury to brain, lack of concentration, perceptual problems, vision, dizziness or vertigo, side effects of medication, all these factors can account for the loss of balance in stroke.

The comparison of post intervention scores of Tinetti-POMA between group $1(18.76 \pm 1.78)$ and group $2(16.8 \pm 1.87)$ showed significant difference $(P=0.018)$. The comparison of postintervention scores of Mini-BESTest between group $1(16.15 \pm 1.28)$ and group $2(14.7 \pm 1.41)$ showed significant difference $(P=0.018)$.

In a study it was found core exercises were given for $30 \mathrm{~min}$ per session, 4 times per week over a period of 5 weeks leading to an increase in the ability in maintaining sitting position (Verheyden et al., 2009). Additionally it was reported that core stability enhancing exercises are effective in improving muscle activities of the lower trunk, which are affected by stroke (Yu and Park, 2013). Ko et al. (2016) investigate an additive effect of core muscle strengthening (CMS) and trunk neuromuscular electrical stimulation (tNMES) on trunk balance in stroke patients. The combination group showed more improvements in Korean version of Berg Balance Scale (K-BBS) and the dynamic sitting balance of TIS, as compared to the CMS group; and more improvement in K-BBS, as compared to the $\mathrm{tNMES}$ group, indicating an additive effect of CMS and $\mathrm{tN}$ MES on the recovery of trunk balance in patients with acute or subacute stroke who have poor sitting balance (Ko et al., 2016).

\section{Improvement in gait scores}

The comparison of postintervention scores of WGS between group $1(20.0 \pm 1.62)$ and group $2(22.01 \pm 2.47)$ showed significant difference ( $t=-2.356, P=0.028)$. Chung et al. (2013) found that core training improves stability of lower trunk and pelvis hence improving static and dynamic balance. Furthermore they found that core training improves stride length, cadence and velocity in stroke participants. Core strengthening improved posterior pelvic tilt and center of gravity transfer during swing phase. It has been proven that transverse abdominis is involved in preparation of body during contralateral weight shifting. Studies show that trunk muscle activity, especially transverse abdominis and multifidus precedes the lower and upper limb muscular activity (Hodges and Richardson, 1997). It is also believed that such feed-forward recruitment pattern of core musculature provides a more stable neuromuscular foundation for muscular movement and can contribute to more precise limb control during locomotion.

There has been improvement in TIS scores. Zakaria et al. (2010) found that weakness of abdominal muscles is accompanied by lat- 
eral trunk deviation. In stroke patients where trunk is a key point in rehabilitation, improving core strengthening along with facilitating the proprioceptors would help to provide better motor control. In another study effect of core stability training on trunk impairment in stroke and found that strengthening abdominal and lumbar muscles help improve selective muscle movements resulting in improved TIS scores (Kim et al., 2015).

There was no statistically significant improvement in the functional ability of the participants. It may be due to the activities measured by BI were not directly related to the primary aim of the intervention.

\section{Clinica message}

The results suggest that there is an additive effect of core strengthening and pelvic PNF for improving trunk impairment, balance, gait, and function in stroke patients. Improved core stability provides a stable base for trunk and leg movements. It can be easily administered as no specific equipment is required.

\section{Limitations of the study}

First, there was no control group without intervention, so it is difficult to exclude effects of the natural recovery process of stroke. Second long-term follow-up assessment was not done hence, future studies are required to evaluate long-term effects. Third, sample size was small in the study and participants were recruited from a specific geographical location. Further studies including a larger patient cohort will be needed to fully verify our results. Fourth sophisticated methods for assessing balance and gait e.g., GAITrite system for temporal and spatial gait parameters and of participants were not used.

\section{CONFLICT OF INTEREST}

No potential conflict of interest relevant to this article was reported.

\section{REFERENCES}

Akuthota V, Ferreiro A, Moore T, Fredericson M. Core stability exercise principles. Curr Sports Med Rep 2008;7:39-44.

Akuthota V, Nadler SF. Core strengthening. Arch Phys Med Rehabil 2004;85(3 Suppl 1):S86-92.

Chung EJ, Kim JH, Lee BH. The effects of core stabilization exercise on dynamic balance and gait function in stroke patients. J Phys Ther Sci 2013;25:803-806.
Hodges PW, Richardson CA. Contraction of the abdominal muscles associated with movement of the lower limb. Phys Ther 1997;77:132-142.

Kibler WB, Press J, Sciascia A. The role of core stability in athletic function. Sports Med 2006;36:189-198.

Kim KY, Chun SP, Kang TG, Kim GD. Effects of core stability training on postural control ability and respiratory function in chronic stroke patients. Adv Sci Technol Lett 2015;88:181-186.

Ko EJ, Chun MH, Kim DY, Yi JH, Kim W, Hong J. The additive effects of core muscle strengthening and trunk NMES on trunk balance in stroke patients. Ann Rehabil Med 2016;40:142-151.

Kumar S, Kumar A, Kaur J. Effect of PNF technique on gait parameters and functional mobility in hemiparetic patients. J Exerc Sci Physiother 2012;8:67-73.

Langhorne P, Bernhardt J, Kwakkel G. Stroke rehabilitation. Lancet 2011; 377:1693-1702.

Mann DK, Raja NR, Bhardwaj N, Singh J. Effect of proprioceptive neuromuscular facilitation in hemiplegic gait a randomized trial of 4 weeks and a follow up after 2 weeks. Indian J Physiother Occup Ther 2013; 7:59-64.

Pathak S, Vijayakumar K, Nayak A, Kedambadi RC. The relationship between pelvic alignment and trunk control in stroke subjects: a crosssectional study. Int J Res Med Sci 2015;2:1483-1487.

Shinde K, Ganvir S. Effectiveness of trunk proprioceptive neuromuscular facilitation techniques after stroke: a meta-analysis. Natl J Med Allied Sci 2014;3:29-34.

Shinkle J, Nesser TW, Demchak TJ, McMannus DM. Effect of core strength on the measure of power in the extremities. J Strength Cond Res 2012;26:373-380.

Taylor CF, Kumar S. Stroke in India factsheet. 2012. New Delhi (India): South Asia Network for Chronic Disease, Public Health Foundation of India; 2012.

Verheyden G, Vereeck L, Truijen S, Troch M, Lafosse C, Saeys W, Leenaerts E, Palinckx A, De Weerdt W. Additional exercises improve trunk performance after stroke: a pilot randomized controlled trial. Neurorehabil Neural Repair 2009;23:281-286.

Wang RY. Effect of proprioceptive neuromuscular facilitation on the gait of patients with hemiplegia of long and short duration. Phys Ther 1994;74:1108-1115.

Yu SH, Park SD. The effects of core stability strength exercise on muscle activity and trunk impairment scale in stroke patients. J Exerc Rehabil 2013;9:362-367.

Zakaria Y, Rashad U, Mohammed R. Assessment of malalignment of trunk and pelvis in stroke patients. Egypt J Neurol Psychiat Neurosurg 2010;47:599-604. 